\title{
Management of Ruptured Scalp Arteriovenous Malformation: A Rare Case and Literature Review
}

\author{
Isaac Okyere 1,2,3*, Sanjeev Singh ${ }^{2,3,4}$, Perditer Okyere ${ }^{2,3,5}$, Paa Kwesi S. Fiifi-Yankson"1, \\ Boutros F. Farhat ${ }^{1}$, Samuel Gyasi Brenu${ }^{1}$ \\ ${ }^{1}$ Department of Surgery, Komfo Anokye Teaching Hospital, Kumasi, Ghana \\ ${ }^{2}$ School of Medicine and Dentistry, College of Health Sciences, Kwame Nkrumah University of Science, Kumasi, Ghana \\ ${ }^{3}$ Komfo Anokye Teaching Hospital, Kumasi, Ghana \\ ${ }^{4}$ Department of Anaesthesia and Intensive Care, Komfo Anokye Teaching Hospital, Kumasi, Ghana \\ ${ }^{5}$ Renal Unit/Department of Medicine, Komfo Anokye Teaching Hospital, Kumasi, Ghana \\ Email: *drokyere@yahoo.com
}

How to cite this paper: Okyere, I., Singh, S., Okyere, P., Fiifi-Yankson, P.K.S., Farhat, B.F. and Brenu, S.G. (2020) Management of Ruptured Scalp Arteriovenous Malformation: A Rare Case and Literature Review. World Journal of Cardiovascular Surgery, 10, 271-278.

https://doi.org/10.4236/wjcs.2020.1012029

Received: June 25, 2020

Accepted: December 27, 2020

Published: December 30, 2020

Copyright $\odot 2020$ by author(s) and Scientific Research Publishing Inc. This work is licensed under the Creative Commons Attribution International License (CC BY 4.0).

http://creativecommons.org/licenses/by/4.0/

\begin{abstract}
Arteriovenous malformation (AVM) are vascular anomalies that arise due to abnormal connection with shunting of blood from arteries to veins without an intervening capillary network. They are mostly found intracranially but extracranially, the scalp AVM cases are also well reported. However, arteriovenous malformation (AVM) of the scalp is a rare lesion whose natural history is not clearly understood. The clinical manifestation of AVM includes asymptomatic state, local discomfort, headaches and occasional scalp necrosis leading to massive haemorrhage. CT or MR angiography remains the cornerstone for investigation. The options of treatment include complete surgical excision, embolization or a combination of the two modalities. We present the successful management of a young man who presented with a torrential bleeding from a ruptured scalp arteriovenous malformation and had to undergo emergency complete excision followed with a rotational flap for the scalp reconstruction.
\end{abstract}

\section{Keywords}

Scalp Arteriovenous Malformation, Bleeding, Surgery, Rotational Flap

\section{Introduction}

Arteriovenous malformation (AVM) of the scalp is a rare vascular lesion. They arise due to abnormal connection with shunting of blood from arteries to veins 
without an intervening capillary network [1] [2] [3]. It is mostly found intracranially but extracranial cases are also well reported [1]. Despite only accounting for $14 \%$ of the total body surface area, about half of all arteriovenous malformations of the skin occur in the scalp [4]. The scalp AVM comprises $8.1 \%$ of total $\mathrm{AVM}$ and is more common in young adults. AVM of the scalp is a rare occurrence particularly when compared to other craniofacial or subcutaneous vascular abnormalities [3]. The high-flow vascular lesion arises from an abnormal arteriovenous connection within the subcutaneous layer of the scalp causing significant aesthetic and psychological complications [4] [5] [6]. The lesion typically consists of one or more dilated feeding arteries with their origins in the dense subcutaneous layer of the scalp, a nidus consisting of a plexus of fistulae, and enlarged and tortuous draining veins [7]. The veins subsequently undergo progressive dilatation and aneurysmal changes that can lead to necrosis of the skin and massive bleeding occasionally with high mortality.

\section{Case Report}

A 28-year-old Ivorian male presented to our facility with a painless, pulsatile and progressively increasing occipital scalp swelling of 8 years duration. He had experienced two episodes of spontaneous bleeding from the swelling previously but they resolved without hospitalization. He had however been referred from his primary care facility in Cote D'Ivoire to Ghana for further management. While on his way to the hospital in Ghana, he had an episode of spontaneous bleeding from the scalp swelling and as such reported to a peripheral hospital where the bleeding was controlled and quickly referred for further management.

On arrival, he had another episode of spontaneous bleeding which was associated with dizziness, palpitations and transient loss of consciousness for which he was immediately resuscitated and transfused with 2 units of whole blood. Haemodynamically, he was fully conscious with blood pressure of $110 / 80 \mathrm{mmHg}$, pulse rate of $95 \mathrm{bpm}$ with moderate volume, respiratory rate of 28 cycles per minute and saturating at $97 \%$ in room air. He had a huge, fluctuant and non-tender occipital mass measuring $15 \mathrm{~cm} \times 20 \mathrm{~cm}$ which was actively bleeding.

Blood chemistry analysis done showed normal parameters while complete blood count reported of haemoglobin level of $9.2 \mathrm{~g} / \mathrm{dl}$, platelet count of $248 \times$ $10^{3} / \mathrm{UL}$ and white cell count of $5.64 \times 10^{3} / \mathrm{UL}$. An accompanying head CT scan taken showed features suggestive of a huge occipital scalp arteriovenous malformation with no evidence of bony malformations or intracranial extension.

Due to the profuse nature of the bleeding, patient was immediately prepared for surgical excision after informed consent. He was put in prone position after general anaesthesia with cuffed endotracheal intubation, then he was prepped and draped and a circumferential incision was made down to the epicranium while securing haemostasis and the mass carefully excised en bloc as shown in Figures 1-4. Rotational flap coverage and split-thickness skin grafting for the scalp defect reconstruction was done by the plastic surgeons on postoperative 
day 12 as shown in Figure 5. Estimated blood loss was approximately $500 \mathrm{mls}$ for the initial emergency excision.

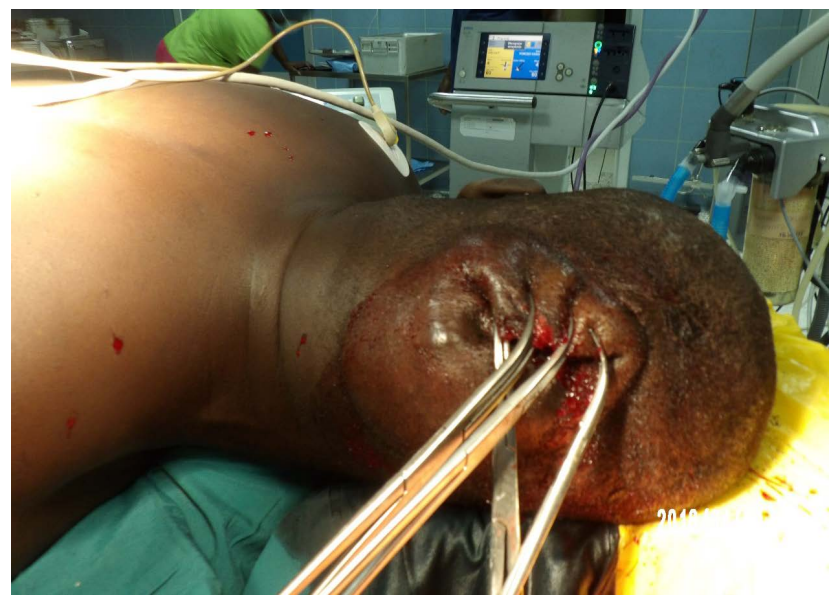

Figure 1. Intraoperative photo under anaesthesia showing the ruptured occipital scalp $\mathrm{AV}$ malformation with controlled bleeding sites.

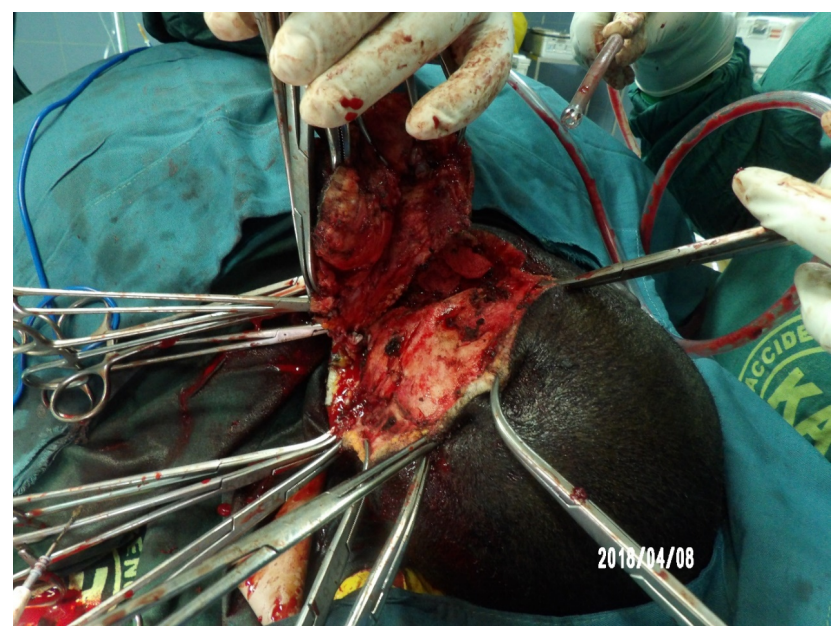

Figure 2. Occipital scalp with nidus excised and elevated.

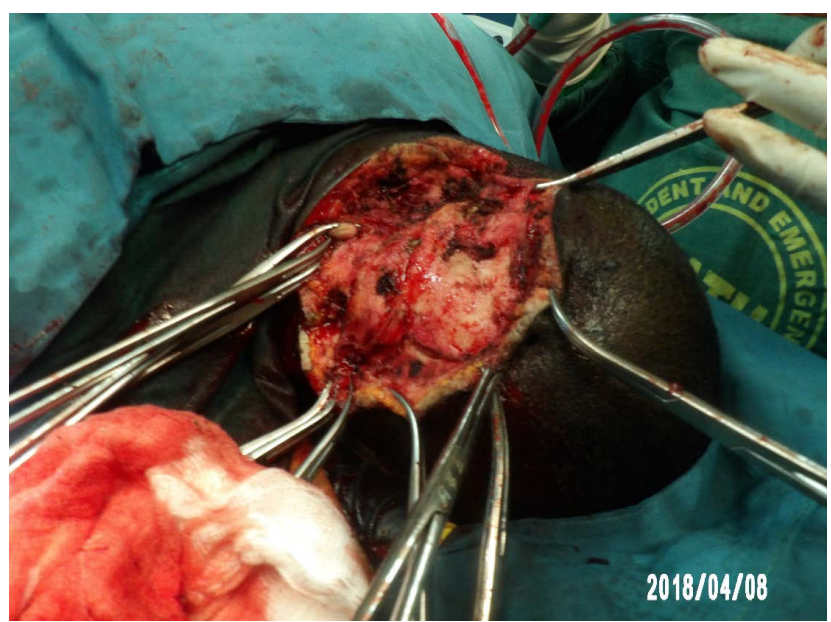

Figure 3. A-V malformation excised en bloc and hemostasis secured. 


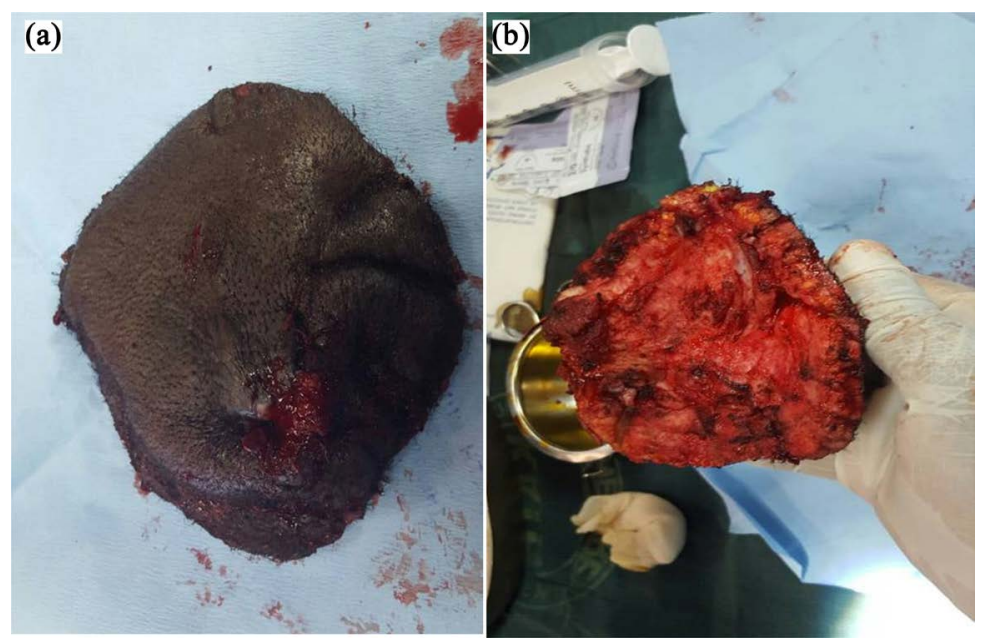

Figure 4. Excised mass for histopathology.
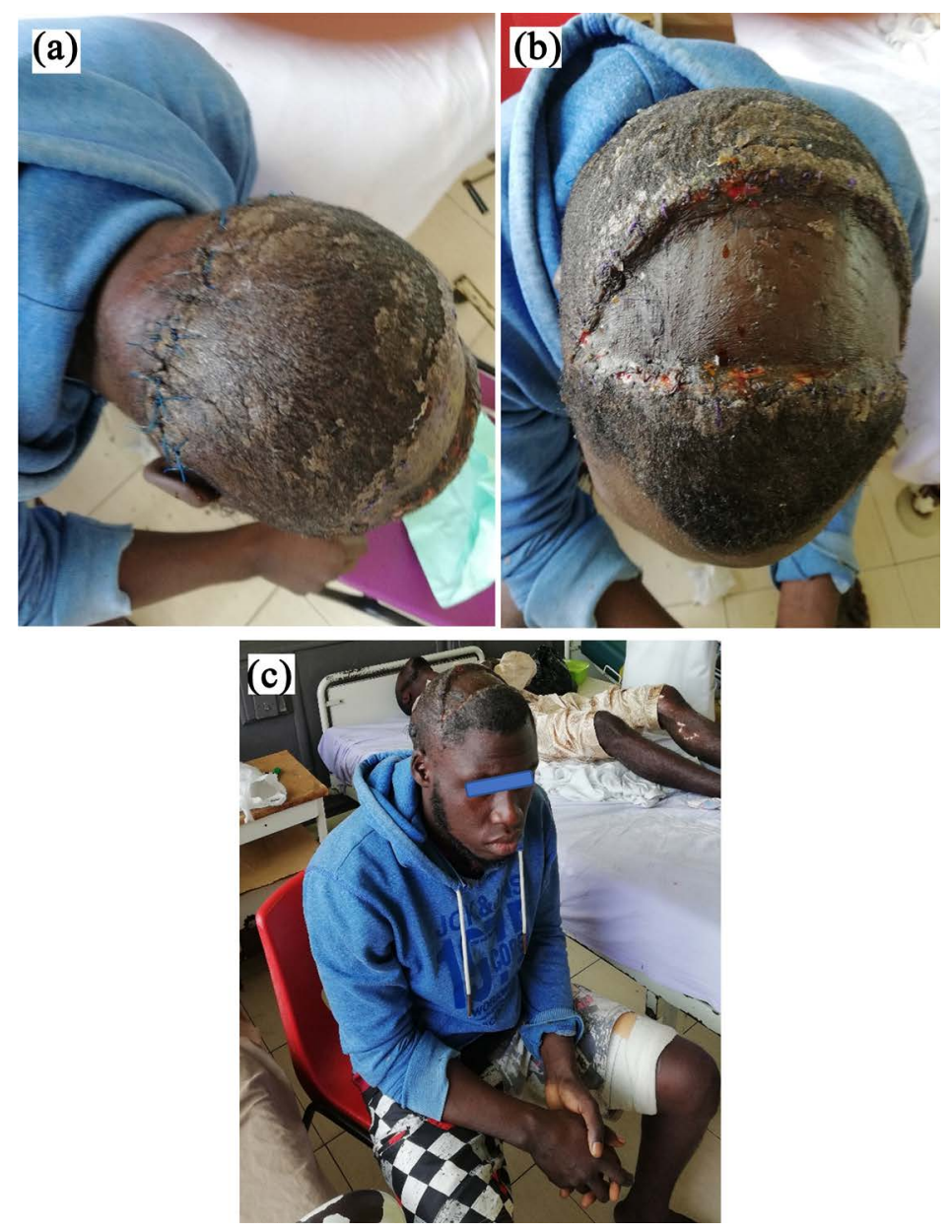

Figure 5. Post-flap coverage and skin grafting of scalp defect.

Patient made a gradual postoperative recovery with excellent take of the graft and flap on the scalp. He was discharged home on postoperative day 23 for follow up on outpatient basis but was lost to follow up because he absconded on discharge. 


\section{Discussion}

Arteriovenous malformations (AVM) are vascular anomalies that arise due to an abnormal connection with shunting of blood from arteries to veins without an intervening capillary network [1] [2] [3]. It is mostly found intracranially but extracranial cases are also well reported [1]. Despite only accounting for $14 \%$ of the total body surface area, about half of all arteriovenous malformations of the skin occur in the scalp [4]. AVM of the scalp is a rare occurrence particularly when compared to other craniofacial or subcutaneous vascular abnormalities [3]. The high-flow vascular lesion arises from an abnormal arteriovenous connection within the subcutaneous layer of the scalp causing significant aesthetic and psychological complications [4] [5] [6]. The lesion typically consists of one or more dilated feeding arteries with their origins in the dense subcutaneous layer of the scalp, a nidus consisting of a plexus of fistulae, and enlarged and tortuous draining veins [7]. The veins subsequently undergo progressive dilatation and aneurysmal changes. Due to its appearance, this condition has over the years acquired several synonyms such as Cirsoid aneurysm, aneurysm serpentinum, aneurysm racemosum, plexiform angioma, aneurysma varix and arteriovenous fistula [1] [4] [5] [6]. Despite being possibly present at birth, scalp AVMs commonly manifest in late childhood, adolescence or early adulthood [3] [5] [6]. However, there have been few reports of infantile cases of scalp AVMs in literature. Chowdhury et al. reported in a case series of 2 cases involving an 8-month old and a 9-month old baby [8]. Hussain and colleagues also published a case report involving a 10-day old baby with a congenital scalp arteriovenous malformation [2]. This is the first experience we have had in the over 50-year history of our hospital and is a young man of 28 years who was referred from a neighbouring country.

Arteriovenous malformations of the scalp frequently occur on the frontal, temporal, occipital and parietal regions of the scalp [1] [4]. Our case was located at the occipital region. Some lesions may be large enough to span multiple regions as was seen by Kumar et al. where almost half of cases reviewed had involvement of more than one contiguous anatomical location [4]. The feeding arteries commonly involved are the superficial temporal, occipital, external carotid and supraorbital arteries [1] [4] [6] [8].

Etiologically, scalp AVMs may be classified as congenital, traumatic, idiopathic or iatrogenic [1] [2] [4] [5] [6] [9]. Congenital scalp AVMs are predominant among the young age population but symptomatic cases are experienced after the second decade of life [6], a finding which fits into our case since the swelling had been noticed for the past 8 years and patient was 28 years at presentation. They arise from an embryologic defect resulting in failure of complete differentiation of primitive vascular structures into arteries and veins. They may present at birth as purple or red birthmarks that may easily be mistaken for capillary hemangiomas [2]. Traumatic AVMs are seen across all the age ranges and account for $10 \%-20 \%$ of all documented scalp AVMs. They tend to develop 
several months to years after the inciting trauma [1]. They are said to arise from formation of a direct connection between the artery and the vein or canalization of a post-trauma thrombus [4]. Iatrogenic scalp AVMs have been reported following procedures such as hair transplantation, craniotomy and vascular access through scalp veins [4] [9]. Stimuli such as pregnancy or puberty, which are associated with significant hormonal changes are known to serve as triggers for exacerbation of the AVMs due to their potential to promote angiogenesis and formation of more collaterals among existing malformations [5] [8] [9].

Based on the origin of the feeding vessels, scalp malformations are also classified as either primary scalp arteriovenous malformations or secondary scalp venous malformations. Primary scalp AVMs have their feeding vessels originating from scalp branches of the external carotid artery and supraorbital artery found within the dense subcutaneous layer of the scalp and are drained by scalp veins. Secondary scalp venous malformations are venous dilatation of the scalp veins due to connection with intracranial vascular malformations [4].

Scalp arteriovenous malformations typically start as small subcutaneous lesions which progress subsequently into huge masses causing disfigurement if left untreated [4] [9]. They may present clinically with a disfiguring and pulsatile mass, headache, tinnitus, localized pain and necrosis of overlying skin [1] [5] [6] [8]. They may also present with episodes of haemorrhage from the mass owing to thin and atrophic overlying skin [3] [4]. Haemorrhage may be so severe as to cause exsanguination, as was experienced by Kumar et al. who reported one patient who died from exsanguinating haemorrhage from a giant scalp AVM [4]. Our case presented with spontaneous bleeding leading to haemorrhagic shock whiles on admission for the second episode of bleeding after he had bled at a peripheral hospital before being referred to our facility. He had to be quickly resuscitated and plan for early surgical excision and haemostasis to save his life. However he had only noticed a painless, pulsatile and progressively increasing occipital scalp swelling of 8 years duration. Chowdhury and colleagues also reported of three patients from their case series with episodes of recurrent scalp AVM haemorrhages [8]. A commonly observed physical finding is the presence of thrill and bruit due to increased flow through the draining veins with turbulence. Advanced stages of the disease, though rare, could present with high output cardiac failure, cerebral steal syndrome and seizures [6].

Based on the clinical progression of the disease, scalp AVMs are described in four stages. Stage I, known as stage of Quiescence is characterized by the presence of pink-bluish stain and warmth over the affected area. The lesion may have similarity with a capillary malformation or an involuting haemangioma. Shunting is only appreciated on Doppler scanning. In Stage II, that is the stage of Expansion, there is an enlarged and pulsatile mass characterized by thrills, bruits and presence of tortuous veins. In the Stage of Destruction (Stage III), there are dystrophic changes on overlying skin with ulceration, bleeding, pain or skin necrosis. Therefore our case presented in the stage III. There may also be associ- 
ated lytic lesions in underlying calvarium. Stage of Decompensation, the final stage is characterized by development of congestive cardiac failure with left ventricular hypertrophy due to the persistent high cardiac output [6].

Despite the diagnosis of scalp AVMs being essentially clinical in majority of cases [4], the use of angiography has been deemed the gold standard for diagnosis [2] [4] [5] [8] [10]. It is invaluable in detecting the angioarchitecture of the lesion and also identifies any associated intracerebral extension or communication [3], a rare but well reported scenario as was seen by Kanth et al. [9]. It could be carried out through the semi-invasive catheter-based approach or non-invasively with the use of computed tomography or magnetic resonance imaging [1] [4] [10]. Doppler ultrasound or brain MRI is also useful in confirmation of diagnosis and for the exclusion of any intracranial communication or origin [4] [6]. Our patient was initially evaluated by CT angiography with confirmation of the AVM before he was referred to our facility.

Options available for treatment of scalp AVMs include surgical excision and percutaneous embolization [2] [5] [8]. Surgical excision has been shown to be the most commonly used and successful option in treatment of complex and scalp lesions. The feeding and draining vessels along with nidus may be carefully dissected out thereby avoiding the need of plastic intervention [5]. However, giant lesions and masses with associated overlying skin atrophy or necrosis may require en bloc resection and scalp reconstruction [4] as was the modality used by our centre for this patient especially after presenting with a bleeding complication in haemorrhagic shock. Success of the surgery depends on the ability to obtain proximal and distal vessel control and comprehensively excise the AVM while providing the best cosmetic result as much as possible as was done for this patient [6].

Advances in endovascular interventions have unearthed the effectiveness of percutaneous embolization of scalp AVMs as a viable treatment option, either as a definitive treatment or preoperative intervention to surgery [4] [8]. The percutaneous approaches for access include transarterial femoral, transvenous femoral and direct catheterization of feeding vessels [5] [8]. Despite reports of its efficiency as a definitive treatment, percutaneous embolization is usually employed as a preoperative adjunct to surgical excision so as to minimize excessive intraoperative blood loss and also buy time for thorough preoperative evaluation [1] [2] [4]. Agents employed in embolization include polyvinyl alcohol, gelfoam, glue and thrombogenic coil [5]. Our patient could have benefited from preoperative percutaneous embolization before complete excision if he had not presented with spontaneous bleeding in haemorrhagic shock.

\section{Conclusion}

Scalp arteriovenous malformation is a rare type of arteriovenous malformation. Treatment options include embolization only, complete surgical excision only, or combination of both embolization and surgical excision with scalp reconstruction. Choices should be based on the size, angioarchitecture, and clinical 
presentations of the AVM. A bleeding scalp arteriovenous malformation is a rare complication of scalp arteriovenous malformation. Aggressive resuscitation, early complete excision and haemostasis save a life.

\section{Acknowledgements}

Written informed consent was obtained from a relative of the patient for the publication and accompanying images.

\section{Conflicts of Interest}

The authors declare no conflicts of interest regarding the publication of this paper.

\section{References}

[1] Hasturk, A., Erten, F. and Ayata, T. (2012) Giant Non-Traumatic Arteriovenous Malformation of the Scalp. Asian Journal of Neurosurgery, 7, 39.

http://www.asianjns.org/text.asp?2012/7/1/39/95698 https://doi.org/10.4103/1793-5482.95698

[2] Hussain, A.S., Ahmed, S.A., Ali, S.R. and Ahmad, K. (2017) Congenital Neonatal Scalp Arteriovenous Malformation: A Very Rare Entity. BMJ Case Reports, 2017. https://doi.org/10.1136/bcr-2016-218756

[3] Mohamed, W.N.Z.W., Abdullah, N.N.L. and Muda, A.S. (2008) Scalp Arteriovenous Malformation: A Case Report. Malaysian Journal of Medical Sciences, 15, 55-57.

[4] Kumar, R., Sharma, G. and Sharma, B.S. (2012) Management of Scalp Arterio-Venous Malformation: Case Series and Review of Literature. British Journal of Neurosurgery, 26, 371-377.

http://www.tandfonline.com/doi/full/10.3109/02688697.2012.654838 https://doi.org/10.3109/02688697.2012.654838

[5] Ozkara, E., Özbek, Z., Özdemir, A. and Arslantaş, A. (2018) Misdiagnosed Case of Scalp Arteriovenous Malformation. Asian Journal of Neurosurgery, 13, 59. https://doi.org/10.4103/1793-5482.181137

[6] Gupta R. and Kayal, A. (2014) Scalp Arteriovenous Malformations in Young. Journal of Pediatric Neurosciences, 9, 263-266. https://doi.org/10.4103/1817-1745.147587

[7] Nagasaka, S., Fukushima, T., Goto, K., Ohjimi, H., Iwabuchi, S. and Maehara, F. (1996) Treatment of Scalp Arteriovenous Malformation. Neurosurgery, 38, 671-677. https://academic.oup.com/neurosurgery/article/38/4/671/2849424 https://doi.org/10.1227/00006123-199604000-00007

[8] Chowdhury, F.H., Haque, M.R., Kawsar, K.A., Sarker, M.H. and Haque, A.F.M.M. (2013) Surgical Management of Scalp Arterio-Venous Malformation and Scalp Venous Malformation: An Experience of Eleven Cases. Indian Journal of Plastic Surgery, 46, 98-107. https://doi.org/10.4103/0970-0358.113723

[9] Kanth, A.M., Ricci, J.A. and Adetayo, O.A. (2019) Diagnosis and Treatment of Scalp Arteriovenous Malformations with Intracranial Extension. Journal of Craniofacial Surgery, 30, 2429-2431. http://journals.lww.com/00001665-201912000-00036 https://doi.org/10.1097/SCS.0000000000005774

[10] Yablonicky, K.J. and Desai, S. (2011) A Case Report of a Scalp Arteriovenous Malformation after Trauma. Journal of Emergency Medicine, 41, e117-e119. https://doi.org/10.1016/j.jemermed.2009.07.039 